\section{Impacto de las condiciones bucales en la calidad de vida de niños y adolescentes}

\author{
Impact of oral conditions on \\ children's and adolescents' \\ quality of life
}

Lucas Guimarães Abreu1,a

${ }^{1}$ Departamento de Odontopediatría y Ortodoncia. Universidad Federal de Minas Gerais, Belo Horizonte, Brasil.

${ }^{a}$ Cirujano Dentista. PhD

Correspondencia:

Lucas Guimarães Abreu

Correo electrónico: lucasgabreu01@gmail.com

Rua Maranhão 1447 / 1101, Bairro Funcionários - Belo Horizonte/ MG Brazil - CEP: 30.150-331

Fecha de recepción: 18/01/18

Fecha de aceptación: 30/01/18
La calidad de vida se ha definido como la percepción de un individuo con relación a su posición en la vida, en el contexto de la cultura y en el sistema de valores en el cual él vive y en relación a sus objetivos, expectativas, patrones y preocupaciones. El constructo calidad de vida posee dos elementos básicos: multidimensionalidad y subjetividad. El primero se refiere a las varias dimensiones o dominios de este constructo, incluyendo los aspectos físicos (síntomas), las funciones, el bienestar emocional y el bienestar social de un individuo. Lo segundo, a su vez, se refiere al hecho que calidad de vida solo puede ser interpretada a partir de la perspectiva del paciente, tomando en consideración percepciones, valores y necesidades. Tales elementos están en consonancia con el concepto de práctica basada en evidencias (PBE) que ha sido ampliamente utilizada en Odontología ${ }^{1}$. La PBE, en Odontología, es un abordaje amplio de cuidados en salud bucal que exige la integración sensata de la experiencia clínica del cirujano dentista con la mejor evidencia científica disponible en la literatura, tomando en consideración la opinión del paciente que está siendo tratado y el de sus familiares ${ }^{2}$.

El concepto de calidad de vida relacionada a la salud bucal (CVRSB) es alusivo a la evaluación de cómo los resultados en salud bucal (enfermedades y sus respectivos tratamientos) impactan la vida y la rutina diaria de los individuos. Desde finales del siglo pasado, la investigación odontológica se ha centrado en la importancia de evaluar la CVRSB y, de esta forma, varios estudios han sido publicados en la literatura científica. Era común que los resultados en salud bucal sean evaluados a través de criterios puramente clínicos con base en las percepciones y el conocimiento de los cirujanos dentistas. Sin embargo, considerar las percepciones del paciente en relación a los resultados en salud bucal en sus propios aspectos físicos, funcionales y también en su bienestar emocional y social se tornó igualmente importante ${ }^{1}$.

Hace algunos años, estudios que evaluaban CVRSB estaban restringidos a poblaciones adultas. Sin embargo, en los últimos 15 años, investigadores se han interesado por el examen de impacto de los resultados en salud bucal en la calidad de vida de los niños y adolescentes. De la misma forma que en los adultos, la evaluación de la CVRSB en Odontopediatría es hecha a través de cuestionarios compuestos por preguntas con el objetivo de medir cuánto las condiciones bucales y sus respectivos tratamientos afectan a la vida de los nińos y adolescentes. Las respuestas para tales preguntas son ordenadas en forma de escalas numéricas.

Dentro de los cuestionarios para el uso de Odontopediatría, podemos mencionar el Early Childhood Oral Health Impact Prolife (ECOHIS), la Scale of Oral Health Outcomes (SOHO), el Child Perceptions Questionnaire (CPQ) y el Oral Health Impact Prolife (OHIP). Los dos primeros son instrumentos usados para evaluar la 
CVRSB en niños preescolares. Se denominan medidas proxy, porque son respondidos por los padres o responsables que ofrecen informaciones relacionadas al impacto de las condiciones bucales en la calidad de vida de sus hijos. El CPQ es un conjunto de cuestionarios para evaluar la CVRSB de nińos y adolescentes. Existen tres instrumentos. $\mathrm{El} \mathrm{CPQ}_{8-10}$ puede ser empleado en evaluaciones de niños entre los 8-10 años. El $\mathrm{CPQ}_{11-14}$ es utilizado en estudios con adolescentes de 11-14 años. El $\mathrm{CPQ}_{11-14}$ puede presentarse en forma larga con 37 preguntas o en su forma corta con un número reducido de preguntas (16). Para evaluaciones en adolescentes de 1519 años, el OHIP es el más adecuado. Esta herramienta también presenta una forma larga con 49 preguntas y una corta con 14 ítems $^{3}$.

Las medidas de CVRSB pueden ser útiles para los odontopediatras y para los encargados de tomar decisiones en salud ${ }^{4}$. Especialistas en la atención de niños y adolescentes, basándose en criterios subjetivos, pueden orientar a sus pacientes y sus padres o responsables con relación al verdadero perjuicio que agravios, tales como caries dental y maloclusión pueden traer para la vida de los individuos jóvenes. La caries dental, por ejemplo, tiene un impacto adverso significativo en la calidad de vida de los niños, generando dolor y síntomas en estos individuos ${ }^{3}$. Las repercusiones negativas de la maloclusión están más relacionadas con los aspectos emocionales y sociales de los jóvenes. Los adolescentes pueden sentirse avergonzados debido a una mala posición de los dientes o debido al traspase horizontal muy exagerado. Esta restricción puede llevar a problemas de socialización y desenvolvimiento con sus pares. Algunos buscan el aislamiento por recelo a que sean excluidos de un grupo de individuos de la misma edad ${ }^{4}$. Los odontopediatras también pueden informar a sus pacientes sobre lo que ellos pueden esperar del tratamiento para enfermedades bucales. Normalmente el tratamiento de caries dental y maloclusión repercuten de forma positiva en la calidad de vida de los nińos y adolescentes. Por ejemplo, individuos con caries dental, cuando son tratados presentan una disminución de los síntomas causados por las lesiones cariosas. El beneficio en la función oral también se hace presente, además de beneficios psicosociales que son significativos.

Responsables de la decisión en salud pueden usar las informaciones de los estudios de CVRSB en la formación de servicios de atención a la población. Muchas veces, los recursos para ser asignados en determinados servicios de atención odontológica son limitados. De esta forma los organizadores de políticas públicas deben de fundamentar bien la asignación de determinados recursos en un sector específico, priorizando los gastos de grandes cantidades para la solución de problemas que realmente incomodan a los pacientes ${ }^{5}$.

\section{Referencias bibliográficas}

1. Allison PJ, Locker D, Feine JS. Quality of life: a dynamic construct. Soc Sci Med. 1997;45(2):221-230.

2. Martins-Júnior PA, Gomez RS, Brennan PA, Abreu LG. Evidence-based dentistry: Challenges and possibilities. J Oral Pathol Med. 2017;46(10):857-858.

3. Gilchrist F, Rodd H, Deery C, Marshman Z. Assessment of the quality of measures of child oral health-related quality of life. BMC Oral Health. 2014;14:40.

4. Abreu LG, Melgaço CA, Bastos Lages EM, Paiva SM. Impact of malocclusion on adolescents' oral health-related quality of life. Gen Dent. 2016;64(6):e1-e5.

5. McGrath C, Broder H, Wilson-Genderson M. Assessing the impact of oral health on the life quality of children: implications for research and practice. Community Dent Oral Epidemiol. 2004;32(2):81-85. 